\title{
2259. Research on fault diagnosis of hydraulic pump using convolutional neural network
}

\author{
Jiyu Yan', Huijie Zhu' ${ }^{2}$, Xiaoqiang Yang ${ }^{3}$, Youhui Cao ${ }^{4}$, Lifu Shao ${ }^{5}$ \\ ${ }^{1,3}$ College of Field Engineering, PLA University of Science and Technology, Nanjing, P. R. China \\ ${ }^{2,4,5}$ The First Engineering Scientific Research Institute, Wuxi, P. R. China \\ ${ }^{3}$ Corresponding author \\ E-mail: ${ }^{1545541212 @ q q . c o m,{ }^{2} q i y u e z h i g u a n g @ g m a i l . c o m,}{ }^{3}$ zhuhuijiehao@163.com, ${ }^{4}$ zhu0126@126.com, \\ 5slfg1998@163.com
}

Received 12 March 2016; received in revised form 22 August 2016; accepted 4 September 2016 DOI https://doi.org/10.21595/jve.2016.16956

\begin{abstract}
The failure mechanism of hydraulic pump is complex, and its faulty features are frequently submerged in the nonlinear interference caused by various components. The fault diagnosis of hydraulic pump is a challenge in the field of machinery. The conventional fault diagnosis approaches have several drawbacks. First, the operator should be cognizant of the mechanism of hydraulic pump. Second, the procedure is onerous, and has many parameters to set. Third, the shallow classification is weak for this complex problem, which leads to low accuracy rate. This paper developed a new scheme by using improved convolutional neural network. It can be directly used without human intervention, although the operator knows little knowledge about hydraulic pump. Therefore, it is simple to be employed and easy for widely promotion. Validated by fault diagnosis cases of hydraulic pump, the proposed scheme is not only simple for application, but also is superior to other machine learning algorithms, especially when the pump speed varies.
\end{abstract}

Keywords: convolutional neural network, fault diagnosis, hydraulic pump, deep learning.

\section{Introduction}

Hydraulic pump is the power source of hydraulic system, its reliable operation directly affects the entire hydraulic system. That is why the hydraulic pump fault diagnosis is a vital task [1]. Hydraulic pump has complex structure, and it usually works continuous in high pressure, so it is prone to failure. A large number of measured data from hydraulic pump show that the faulty features are usually submerged in interference, and the faulty features are often fuzzy. For the reason that traditional diagnosis methods are difficult to detect the failures of hydraulic pump, this problem has been a challenge in the field of industry, thus the research on hydraulic pump fault diagnosis is of great significance.

Traditional fault diagnosis approaches are generally based on mechanism, characteristic frequency or faulty feature extraction [2]. The signal of faulty hydraulic pump is a union of machinery and hydraulic, so its model and feature are difficult to acquire. In addition, although the hydraulic pump is failure for different reasons, it may generate the same fault characteristic frequency. For the purpose of raising the fault diagnosis accuracy rate for hydraulic pump, a lot of scholars have done many fruitful researches. Du [3] analyzed the statistics of the characteristics frequency measured from hydraulic pump, and calculated the failure threshold by experience, in order to diagnose the multiple faults of hydraulic pump. Gao [4] used wavelet packet coefficient and residual of the signal energy for fault recognition of hydraulic pump, and he also set threshold through the experience. Dong [5] established the hidden semi-Markov models for hydraulic pump using vibration signals under different conditions, in order to recognize the failure. Du Jingyi [6] extracted the statistics of the vibration signal for fault recognition hydraulic pump.

Although these diagnostic approaches have played a huge role in the development of the fault diagnosis for hydraulic pump, there are still some drawbacks. First, the comprehensive analysis of failure mechanism should be taken, in order to extract significant features. Second, the onerous procedure as well as so many parameters result in difficulties in application. Third, the accuracy rate of existing diagnostic approaches is still not high enough, especially when the operating 
condition is complex, such as the early fault under variable speed. Forth, the popularization is difficult. For a new type pump, the mechanism should be researched again, as well as the feature extraction, feature selection, and new parameters should be tried several times.

Therefore, we hope to exploit a new diagnostic scheme. First, it could be used by a green hand, although he knows little about the fault mechanism of the complex hydraulic pump. We also hope it is simple, and complicated feature extraction and feature selection are automatic by computer. What's more, we hope the accuracy rate is high enough even though the operating condition is bad. Finally, this scheme is easily promoted to a new machine without numerous settings.

The deep learning algorithms - convolutional neural network $(\mathrm{CNN})$ has potential for this diagnostic scheme. It has attracted widespread interest in recent years [7], and it has powerful self-learning and modeling capabilities. CNN has made breakthroughs in the fields of image recognition [8], voice processing [9] and handwritten digit recognition [10] and so on. Although CNN's unique topology structure makes it be highly invariant to translation, scaling, which matches the characteristic of hydraulic pump vibration signal time in time domain [11], it cannot work well in this task. This paper makes some breakthoughes to solve these problems. This scheme directly uses the original data measured from vibration sensor, and it takes the modeling and recognizing process completely to $\mathrm{CNN}$, which doesn't demand for a prior diagnostic knowledge of the operator. In addition, according to the characteristics of vibration signal for hydraulic pump, this paper puts forward a kind of empirical network setting method, and it further reduces the requirement for the operator. By comparison with other intelligent algorithms, this approach is the easiest one and has highest recognition accuracy rate, especially when the speed varies.

\section{The theory of $\mathrm{CNN}$ for hydraulic pump diagnosis}

Deep learning has achieved a breakthrough progress, and it broke the best records in many fields, making the history of the highest accuracy rate [12]. Deep learning mainly contains three models: stacked autoencoders (SAE), deep belief network (DBN) and CNN. The vibration signal of hydraulic pump is period, so its features are of strong translation, which makes DBN and SAE cannot directly process the vibration signal $[13,14]$. Among them, $\mathrm{CNN}$ is shift invariant to local features and its unique weight sharing mechanism suits the structure of image and sound signal. However, the periodic vibration signal of hydraulic pump is special. CNN is improved so that it can extract the invariant features, the section below will introduce the theory of improved CNN.

\subsection{The theory of $\mathrm{CNN}$}

$\mathrm{CNN}$ is a feed-forward neural network, and it extracts topology from a two-dimensional image through back-propagation algorithm to optimize the network, in order to compute the nodes in the network [15]. The introduction of one-dimensional CNN network is followed.

\subsubsection{The structure of $\mathrm{CNN}$}

Fig. 1 is a typical 6 layer $\mathrm{CNN}$, including input layer, output layer, convolution layer, subsampling layer and full connection layer. One dimensional signal is input into input layer, through repeated subsampling layer (S), convolution layer (C) and the last full connection layer (F), finally, the output layer gives the label.

Both the $\mathrm{S}$ and $\mathrm{C}$ have several feature maps, and each map connects with the former layer. At a convolution layer, the previous layer's feature maps are convolved with learnable kernels and input a activation function to constitute the output feature map.

In Fig. 1, the convolution layer are $\mathrm{C} 2$ and $\mathrm{C} 4$, and followed by $\mathrm{S}$. Each feature map in a convolution layer may connect to several feature maps in previous layer. The input signal is $\mathbf{x}_{j}^{l} \in \mathbb{R}^{n \times 1}$, indicates the number of layer, $j$ is number. The convolution kernel is a column vector 
$\mathbf{k}_{i j}^{l} . i$ is the number of feature map in convolution layer. The convolution layer can be represented as:

$\mathbf{x}_{j}^{l}=f\left(\mathbf{z}^{l}\right), \quad \mathbf{z}^{l}=\sum_{i \in M_{j}} \mathbf{x}_{i}^{l-1} * \mathbf{k}_{i j}^{l}+b_{j}^{l}$.

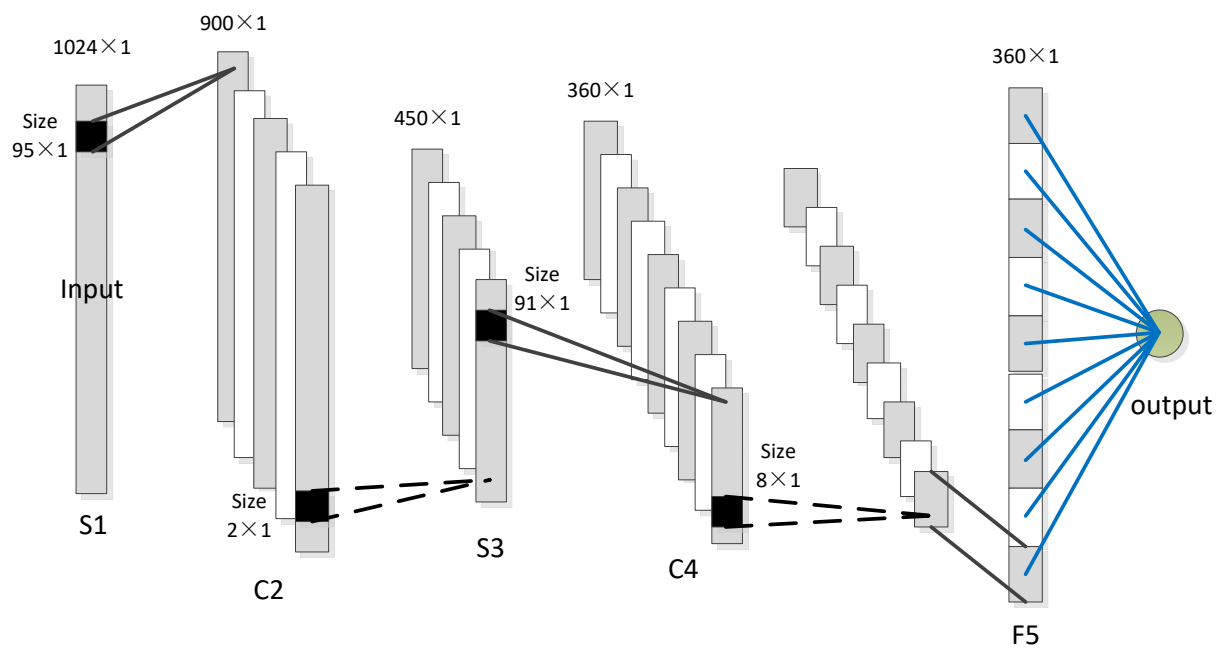

Fig. 1. One dimension CNN

Each feature map corresponds several convolution kernels. $M_{j}$ is a selection of input feature map, here the full connection is chosen, thus each convolution layer has a bias. The main task of convolution layer is to translate former layer in different perspective for constructing model. The convolution layer is usually followed by a subsampling layer:

$x_{j}^{l}=\operatorname{down}\left(x_{i}^{l-1}\right)=\max x_{i}^{l-1}\left(u_{1}, u_{2}\right)$,

where $\left(u_{1}, u_{2}\right)$ represents the pooling scale in previous layer. The purpose of pooling is to reduce resolution and improve computational efficiency, at the same time to enhance the robustness to scaling and distortion. Many experiments have shown this simple way is very effective.

\subsection{Computing $\mathrm{CNN}$}

CNN needs to be computed after, and if its parameters are updated as traditional neural network, each connection will have a parameter which should to be learned. In this condition, the parameters will be too numerous to update. The weight sharing adopted by CNN can greatly reduce the free parameters through sharing the same weight in the same feature map, which can detect the different transform from different perspective using the same kernel.

As shown in Fig. 1, the neuron in layer $\mathrm{C}$ is calculated by the area connected to it. Similarly, the neuron in layer S should be computed by the local field connected to it in previous layer, in this way, the feature map is constructed. In CNN, any neuron in each layer is computed through the local field in previous layer connected to it after a output activation function. Finally, the output layer outputs result, namely the condition of the pump is recognized.

In $\mathrm{CNN}$, weight update is based on the back-propagation algorithm, the update of weight $w$ from iteration $t$ to $t+1$ is as followed:

$w(t+1)=w(t)+\eta \delta(t) x(t)$ 
where $\eta$ is learning rate, $x(t)$ is the input of neurons. $\delta(t)$ is the error term of the neuron, the error term $\delta(t)$ have different expression for output neurons and hidden neurons, and it is usually computed by gradient descent method. References [11, 16, 17] give the details of weight update in $\mathrm{CNN}$.

\subsection{Weighted sum}

As showed in proposed CNN structure, in the last layer namely the full connection layer, the neurons in the same feature map still contains relative position information, thus the translation cannot be completely eliminated. To overcome this challenge, a weighted sum strategy is added to further improve the shift-invariance of CNN. As shown in Fig. 2, a new layer is inserted between the full connection layer and output layer. This new layer sums up the neurons in the same feature map in order to remove the influence of relative position information. This algorithm reduces the neurons used for recognition and also decreases the complexity of network, and it is easy to use and understand. Although it is simple, it indeed effectively enhances the robustness of CNN to translation, and lastly enhances the recognition effect.

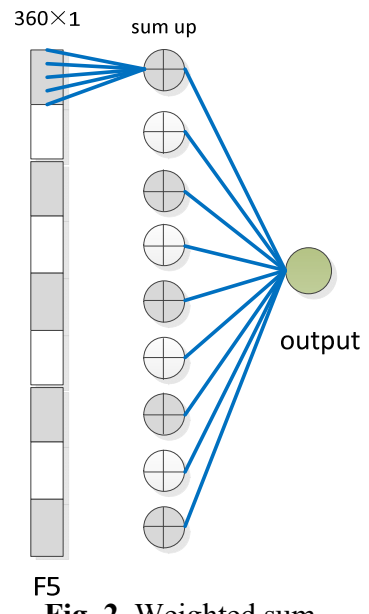

Fig. 2. Weighted sum

\subsection{Diagnosis procedure}

The traditional fault recognition model is as shown in Fig. 3. Firstly, complex pre-process is applied to filter the noise and reduce the influence of variable conditions. Then is feature extraction and selection which should be on the foundation of adequate professional knowledge. The operator's quality is highly demanding in this step, and whether the extracted feature is good enough mainly determines the accuracy of fault diagnosis later. Finally, a classifier is used for fault recognition, and the diagnostic result is acquired. The primary improvement of CNN classification model is that it directly learns the signals measured from sensor without other processing, as displayed in Fig. 4, which considerably reduces the steps. This new model relies on the robustness of CNN to interference, distortion, scaling. It uses CNN to construct the model by the data itself, and automatically finds the internal structures of original data.

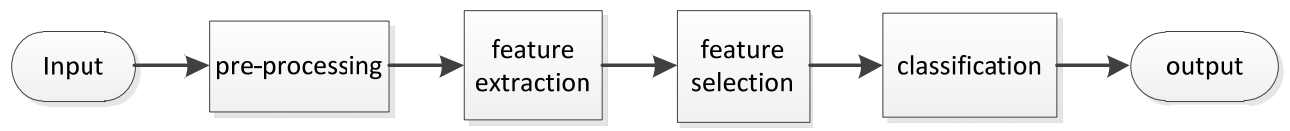

Fig. 3. Traditional classification model 


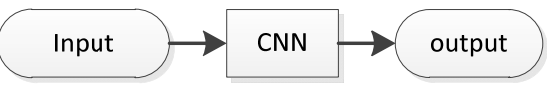

Fig. 4. CNN classification model

\section{Experiment and analysis}

\subsection{Introduction of experiment}

MCY14-1B axial piston pump is small, light, compact and convenient for maintenance, and it is suitable for the machinery in ship, aviation, mining and so on. The type of the hydraulic pump in this experiment is $25 \mathrm{MCY} 14-1 \mathrm{~B}$, and it has 7 plunger. Its nominal flow-rate is $25 \mathrm{ml} / \mathrm{r}$, and the nominal pressure of $31.5 \mathrm{MPa}$.

The hydraulic pump was driven by a three-phase asynchronous motor of $5.5 \mathrm{~kW}$, and the motor was controlled by a frequency converter. The outlet of the hydraulic pump connected a throttle valve and a relief valve, and finally linked to the tank. In this experiment, the load was imposed by the throttle valve, and relief valve kept safety, with the maximum relief pressure was $16 \mathrm{MPa}$. The vibration signal of the hydraulic pump was measured for fault diagnosis, and its sampling frequency is $10 \mathrm{kHz}$. The test rig is as shown in Fig. 5, and the schematic diagram is shown in Fig. 6.

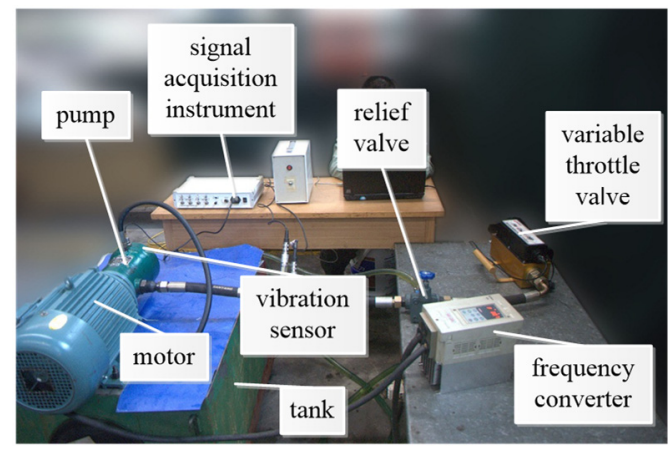

Fig. 5. Test rig

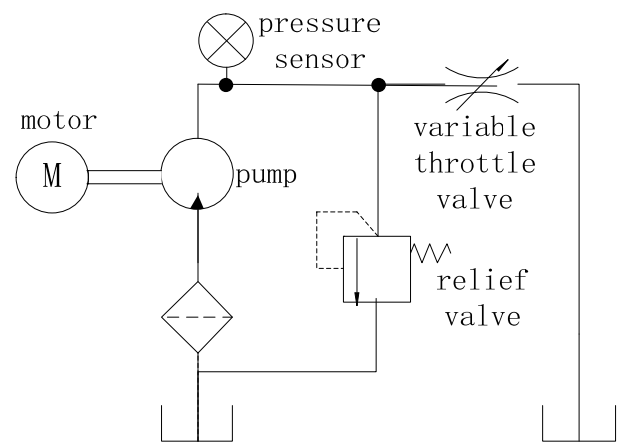

Fig. 6. The schematic diagram of the test system

The axial piston pump's pressure is high and its speed is fast, so it is prone to failure. According to statistics [3], the easiest failure components include cylinder, valve plate, piston, rolling and spring, etc. Six kinds of faults were simulated in this paper, as shown in Fig. 7.

Fault 1: cylinder wear, here cylinder was equivalent to an inner ring of bearing, and the fault was cut as depth of $0.5 \mathrm{~mm}$, width of $0.2 \mathrm{~mm}$.

Fault 2: valve plate wear, the faulty valve plate was chosen in a faulty pump.

Fault 3: roller wear, the fault was cut as depth of $0.5 \mathrm{~mm}$, width of $0.3 \mathrm{~mm}$.

Fault 4: loose slipper, the gap of piston was $0.25 \mathrm{~mm}$.

Fault 5: sliding boot wear, its wear loss is about $0.25 \mathrm{~mm}$ compared to normal one.

Fault 6: spring wear, the wear loss of spring was about $0.25 \mathrm{~mm}$.
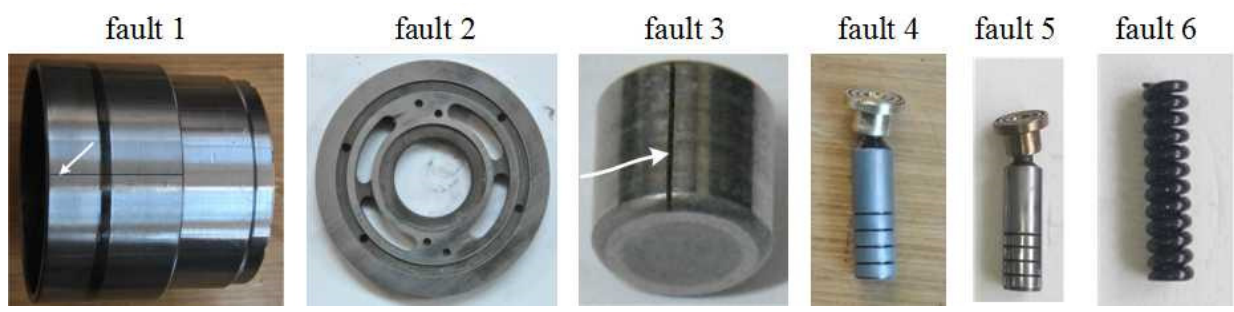

Fig. 7. The faults setting 
The pump speed was $600 \mathrm{r} / \mathrm{min}, 900 \mathrm{r} / \mathrm{min}$ and $1200 \mathrm{r} / \mathrm{min}$ respectively in the experiment, so the rotating frequency was $15 \mathrm{~Hz}$ at the speed of $900 \mathrm{r} / \mathrm{min}$, and the fundamental frequency of pressure pulsation was $7 \times 15=105 \mathrm{~Hz}$. The diameter of the outer ring of the bearing is $107.5 \mathrm{~mm}$, and the diameter of inner ring namely the cylinder is $96.5 \mathrm{~mm}$. The number of rollers is 22 , and its diameter is $11 \mathrm{~mm}$. The outer ring is fixed when pump is working. Therefore, the characteristic frequency of outer ring is about $148.12 \mathrm{~Hz}$, the roller is about $146.45 \mathrm{~Hz}$, and the cylinder (or inner) is about $181.89 \mathrm{~Hz}$.
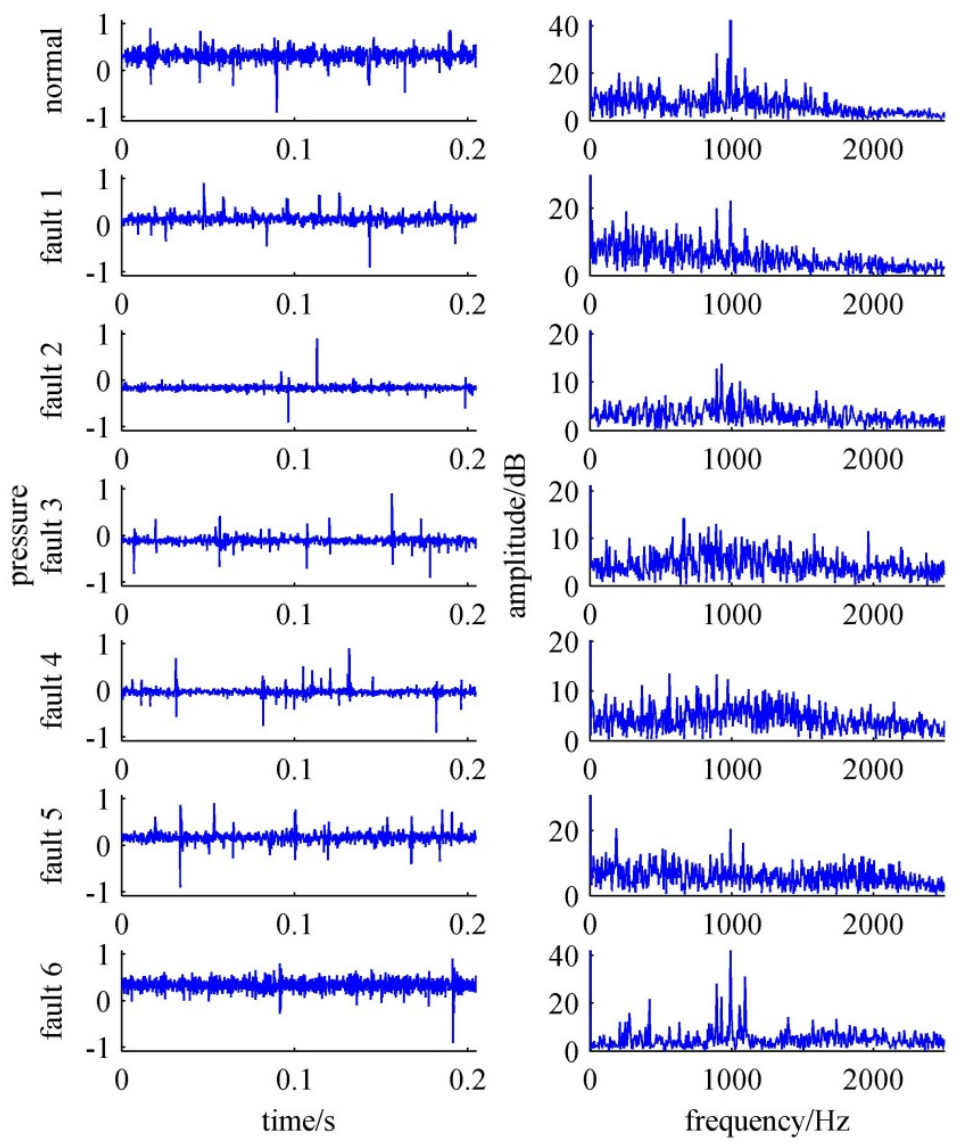

Fig. 8. Vibration signal in time domain and frequency domain of each condition

The hydraulic pump which is complex has many vibration sources. The faulty pump not only changes at characteristics frequency, but also at rotating frequency and natural frequency. Therefore, if we use the characteristic frequency for fault recognition, it will be not only difficult to identify the pump condition, but also losses some potential information. Especially for slight failure with heavy disturbance, it adds more difficulties. Fig. 8 is the vibration signal in time domain and frequency domain of each condition. Although it was under lab experiment, the surrounding noises as well as electromagnetic interference were not removed, thus it was more close to real fault diagnosis. The electromagnetic interference is obvious in each vibration signals. The vibration signal of hydraulic pump is complicated and contains lots of noises. It is nonlinear, leading to difficulties to extract the features which can reflects the status information of the hydraulic pump. From those we can know that, the traditional diagnostic approaches are not easy to diagnose conveniently. Therefore, the following researched how to apply CNN for correct and convenient fault diagnosis of hydraulic pump. 


\subsection{Fault diagnosis and comparison under the stable operating condition}

This section employed proposed method to diagnose the hydraulic pump on the stable speed of $900 \mathrm{r} / \mathrm{m}$. For simple network setting, according to the mechanism of hydraulic pump, an convenient network setting method based on experience was put forward. The CNN in this experiment has 7 layers, as shown in Fig. 9. The first layer is input layer, the 6th layer is full connection layer, and the 7 th is the weighted sum layer. The 2 th and 4 th layer are both convolution layers, and the size of a kernel approximately corresponds a fundamental frequency, here it is termed as basic period. Because hydraulic pump is a rotating machinery, the vibration waveforms in a basic period under different conditions have significant differences, and 2 th layer is used to extract and recognize these differences.

After the subsampled with scale of 3 th in third layer, although the convolution kernel scale still corresponds to a basic period in layer 4, it corresponds to two basic period of the input in actual. Therefore, this kernel can also detect the variation of basic period caused by condition changes. The 2 th layer was used to extract local information, and the 4 th layer was to extract more comprehensive information. This network designing scheme is a good and convenient one validated in experiments, and it also effects for rotating machinery.

Layer 3 and layer 5 are both subsampling layer, the subsampling scale in layer 5 can be a larger one. In this way, it can not only reduce the number of network nodes, but also suppresses the influence of noises and enhances the robustness. At the $\mathrm{CNN}$ of this experiment, the 2 th layer has 8 feature maps, and the 4 th has 16 ones. The scale of each feature map is as displayed in Fig. 9. The activation function is a tanh-function, and output function is a softmax classifier.

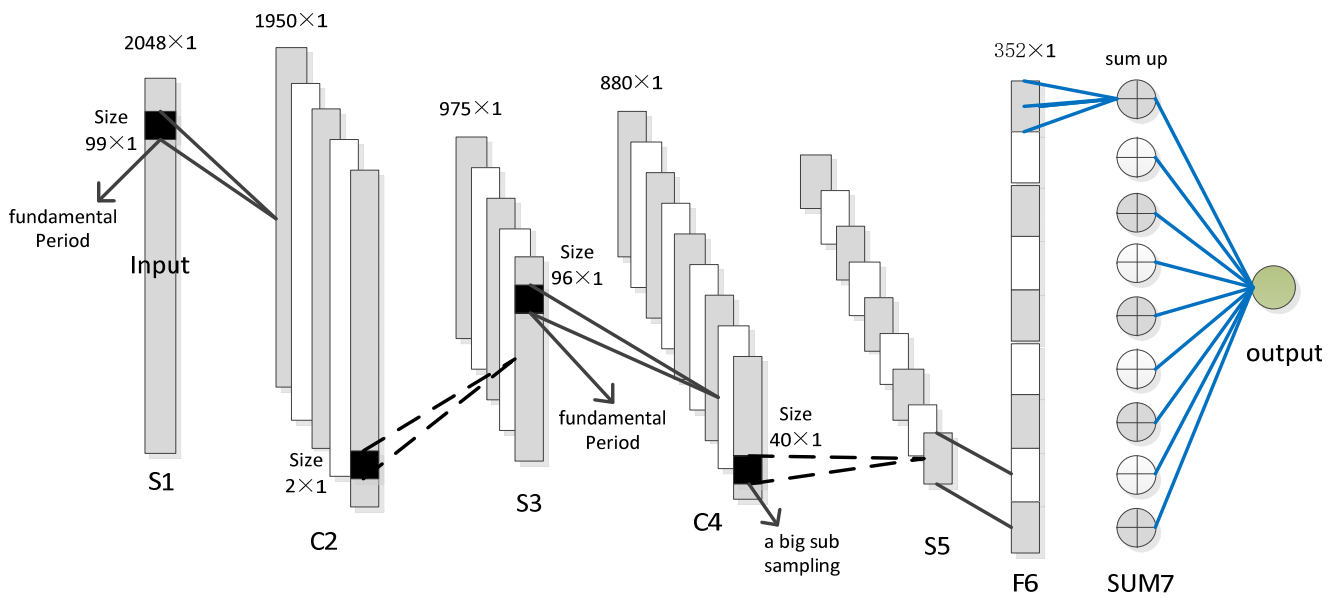

Fig. 9. The CNN structure in experiment

In order to completely verify the fault diagnosis performance by $\mathrm{CNN}$, it was also compared with DBN, support vector machine (SVM) and BP neural network. However, these three algorithms cannot keep shift-invariance, so all the samples were converted to the spectrum for fault recognition. Each class of training set had 100 samples, and each class of test set had 300 samples.

The result is represented in Fig. 10, all the results are the average for ten times of experiments. The accuracy rate of CNN is the highest. Apart from accuracy rate, only CNN directly applies the original data without any additional steps, whereas other methods need attached pre-processing such as time-frequency transform and de-noising. Overall, all of these algorithms work well in this experiment, and the worst one has the accuracy rate above $95 \%$. This is because the speed was stable, so the training samples are similar to the test samples, so even simple algorithms can do this diagnosis. However, the speed of the pump in application cannot always works in such 
stable condition, so the fault diagnosis of variable speed has more engineering significance.

Furthermore, the original CNN without weighted sum was also tested, to verify the improved strategies. The training samples are chosen as 50,100,150,200,250,300, respectively. The result is as shown in Figure 11. The CNN proposed in this paper is superior to original CNN. As the training samples become more, the original CNN performs better. Because the features extracted by the original CNN still have certain position information, when the training samples are few, it is difficult to distinct different condition of hydraulic pump. However, as the number of training samples grows, the shortage will be covered in a certain degree. The improved CNN is invariant to period features, thus it works well although the training samples are few. This experiment further demonstrates that the weighted sum is proper for the hydraulic pump.

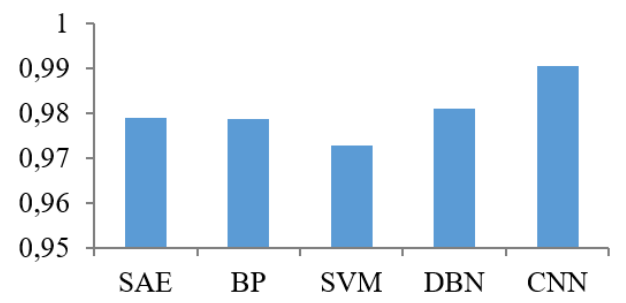

Fig. 10. The accuracy rate for stable working condition

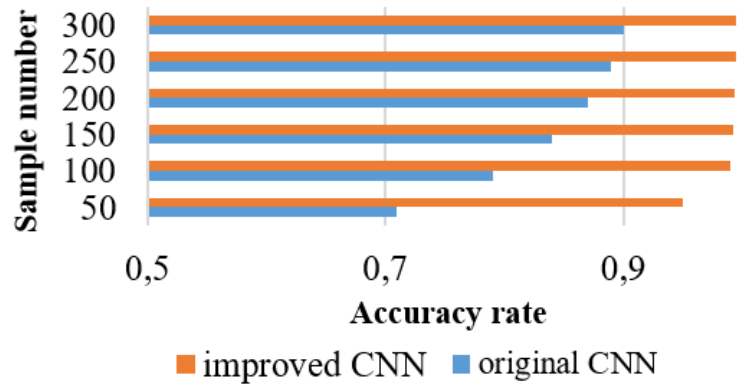

Fig. 11. The accuracy rate of original $\mathrm{CNN}$ and improved $\mathrm{CNN}$

\subsection{Fault diagnosis and comparison under the condition of variable speed}

This experiment was the fault diagnosis of hydraulic pump with variable speed, and other algorithms were also compared. Here the training samples were taken from the speed of $600 \mathrm{r} / \mathrm{m}$ and $1200 \mathrm{r} / \mathrm{m}$, and the test samples are from $600 \mathrm{r} / \mathrm{m}, 900 \mathrm{r} / \mathrm{m}$ and $1200 \mathrm{r} / \mathrm{m}$. The recognition result is shown in Fig. 12, and Fig. 13 is the accuracy rate of each state.

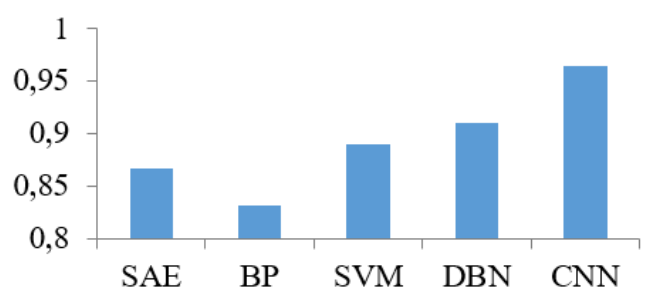

Fig. 12. The accuracy rate for variable speed

It is known from the fault recognition result, the accuracy rate of $\mathrm{CNN}$ is the highest, and the worst one is above $90 \%$. The accuracy rate of CNN is close to the accuracy rate of stable speed, yet the other methods degrade sharply. Due to excellent robustness to scaling, CNN is not sensitive to the variation of speed, so that it is appropriate to the real fault diagnosis of hydraulic pump in 
application. When the speed varies, the frequency spectrum of vibration signal will changes correspondingly, so the other machine learning algorithms were influenced. On the other hand, if the other machine learning algorithms directly used the time domain signal for fault recognition, the performance will be worse. Although DBN is one of deep learning algorithm, it is weak robust to scale. Thus, its fault recognition is not good enough, and its accuracy is the second. The shallow learning method such as SVM and BP neural network perform worse because they are short of the ability to learn from complex data. Due to the actual significance of fault diagnosis with variable speed, this experiment illustrates that the proposed CNN algorithm is applicable to the fault diagnosis of hydraulic pump even the speed is variable.

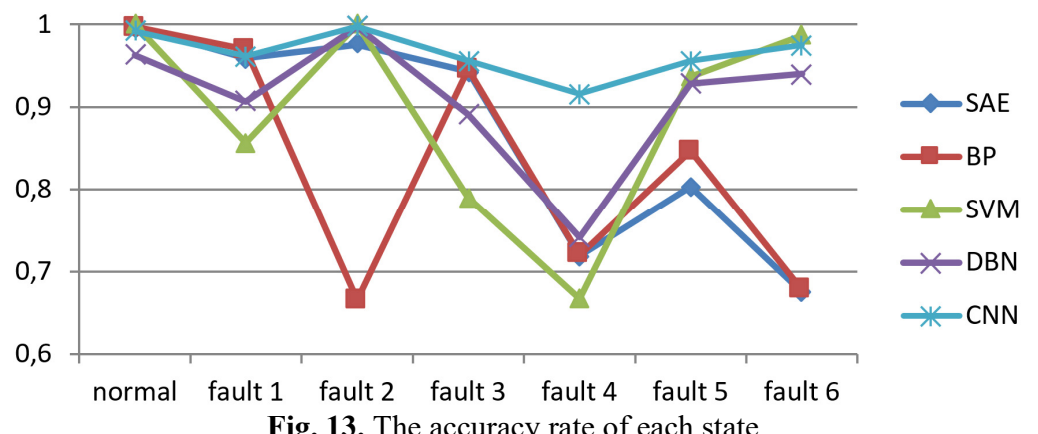

\subsection{The fault separation of hydraulic pump}

Fig. 14 is a hydraulic pressure station, it supplies a driven system. In August 2015, the strength of driving system was too weak to work, and the faulty place is unknown. Possible part is pump, valve or actuator. Firstly, the pump was tested, and it was installed on the test rig to measure the vibration signal. The parameters in this experiment were the same as section 3.3, only the test speed was set as $900 \mathrm{rpm}$. Ten samples were tested, and 9 samples showed normal and one was as sliding boot wear. The procedure of one normal sample put in improved CNN is displayed in Fig. 15. Therefore, the diagnosis of normal is reliable, then it is found that the actuator was failure.

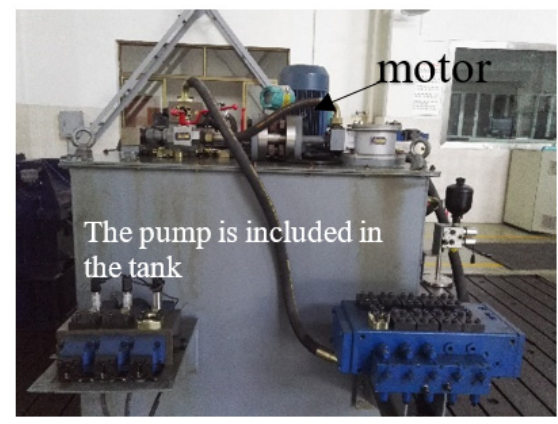

Fig. 14. Hydraulic pressure station

In April 2016, the output power of system was insufficient, and the noise of the hydraulic pump increased when the pressure was high. The hydraulic pump was measured another time and ten samples were measured. Eight samples showed loose slipper, and one as roller wear, another one as valve plate wear. Then the hydraulic pump was disassembled and showed loose slipper. After the replacement of all the plungers, the system worked well again.

The application showed that this scheme can guide the fault diagnosis of hydraulic pump to a certain extent. In the future, the hydraulic pump will be tested under working without disassembling, and the real-time monitoring of the fault diagnosis will also be tried. 

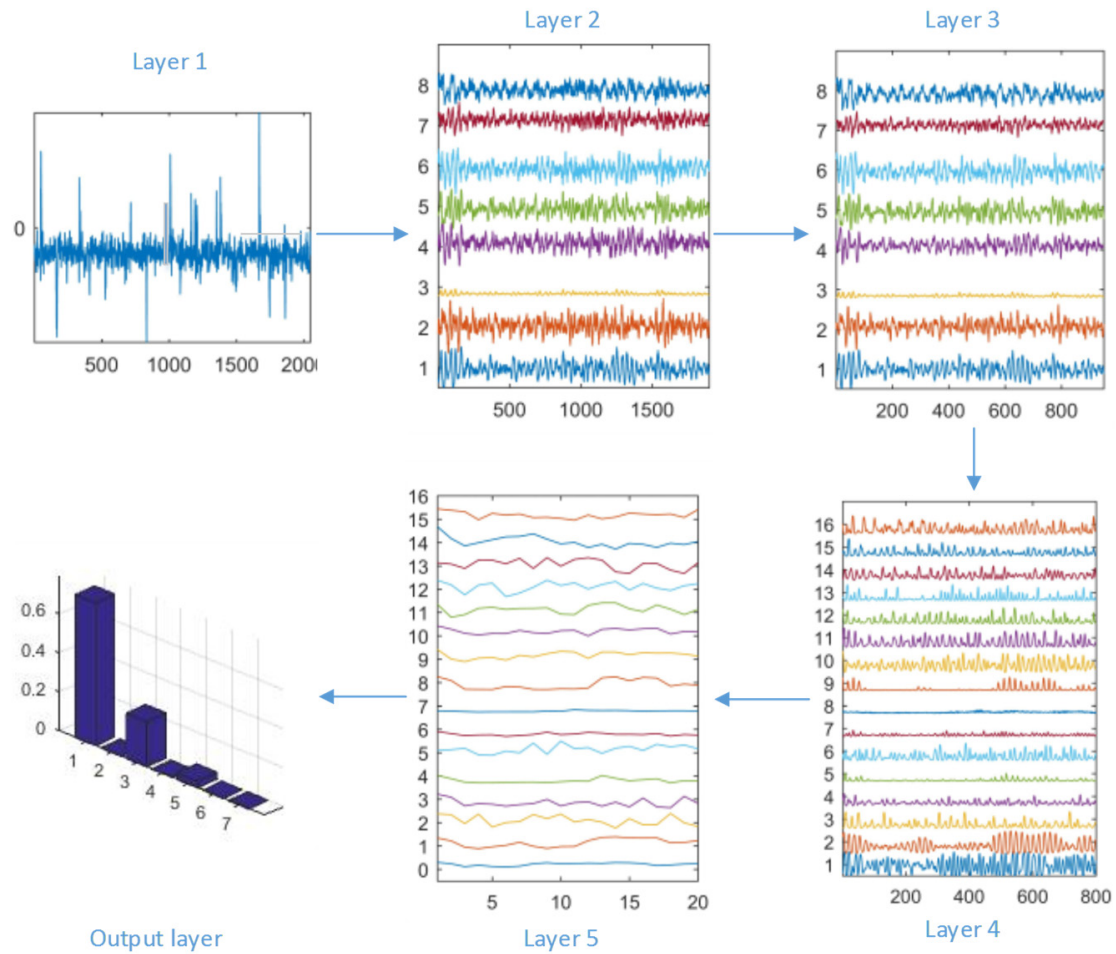

Output layer

Fig. 15. Hydraulic pressure station

Jiyu Yan conceived the work that led to the submission, contributed significantly to analysis and manuscript preparation; Huijie Zhu designed the work and acquired data, and played an important role in interpreting the results; Xiaoqiang Yang approved the final version; Lifu Shao and Youhui Cai helped perform the analysis with constructive discussions.

\section{Conclusions}

Hydraulic pump is easily failure, but due to complex structure, it is very difficult to recognize the fault. This paper improved CNN for the fault diagnosis of hydraulic pump, and proposed an empirical network designing method according to the character of hydraulic pump. CNN has powerful capacity for modeling only using the original data, and it is robust to translation, distortion and scaling. Therefore, the original signal measured from sensor was directly applied to diagnose the pump without tedious pre-treatment or feature extraction steps, and the main task was handed over to the computer, which considerably reduces the difficulty in practical application. Compared to other machine learning algorithms, CNN has the highest accuracy rate for fault identification. Although the speed varies, it can still perform excellently. Consequently, the mentioned algorithm based on CNN is applicable to the fault diagnosis of hydraulic pump, and it has great application value in engineering.

\section{References}

[1] Jiqiao M. A., Hongru L. I., Baohua X. U., et al. Fault model identification of hydraulic pump based on mlse and extension theory. Clinical Radiology, Vol. 64, Issue 3, 2015, p. 256-264.

[2] Tian Y., Lu C., Wang Z. L. Approach for hydraulic pump fault diagnosis based on wpt-svd and svm. Applied Mechanics and Materials, Vols. 764-765, 2015, p. 191-197.

[3] Du J., Wang S., Zhang H. Layered clustering multi-fault diagnosis for hydraulic piston pump. Mechanical Systems and Signal Processing, Vol. 36, Issue 2, 2013, p. 487-504. 
[4] Gao Y., Zhang Q. A wavelet packet and residual analysis based method for hydraulic pump health diagnosis. Proceedings of the Institution of Mechanical Engineers, Part D: Journal of Automobile Engineering, Vol. 220, Issue 6, 2006, p. 735-745.

[5] Dong M., He D. A segmental hidden semi-markov model (hsmm)-based diagnostics and prognostics framework and methodology. Mechanical Systems and Signal Processing, Vol. 21, Issue 5, 2007, p. 2248-2266.

[6] Du J., Hou Y. Online fault early warning for hydraulic pump based on support vector machine. Journal of China Coal Society, Vol. 31, Issue 5, 2006, p. 684-688.

[7] LeCun Y., Bottou L., Bengio Y., et al. Gradient-based learning applied to document recognition. Proceedings of the IEEE, Vol. 86, Issue 11, 1998, p. 2278-2324.

[8] Ciresan D., Meier U., Schmidhuber J. Multi-column deep neural networks for image classification. IEEE Conference on Computer Vision and Pattern Recognition (CVPR), 2012.

[9] Abdel-Hamid O., Mohamed A.-R., Jiang H., et al. Applying convolutional neural networks concepts to hybrid nn-hmm model for speech recognition. IEEE International Conference on Acoustics, Speech and Signal Processing (ICASSP),2012.

[10] Sermanet P., Chintala S., LeCun Y. Convolutional neural networks applied to house numbers digit classification. 21st International Conference on Pattern Recognition (ICPR), 2012.

[11] Lee H., Grosse R., Ranganath R., et al. Convolutional deep belief networks for scalable unsupervised learning of hierarchical representations. Proceedings of the 26th Annual International Conference on Machine Learning, 2009.

[12] Deng L., Li J., Huang J.-T., et al. Recent advances in deep learning for speech research at Microsoft. IEEE International Conference on Acoustics, Speech and Signal Processing (ICASSP), 2013.

[13] Wang X., Zhu H., Rui T., et al. Shift invariant sparse coding ensemble and its application in rolling bearing fault diagnosis. Journal of Vibroengineering, Vol. 17, Issue 4, 2015, p. 1837-1848.

[14] Zhu H., Wang X., Zhao Y., et al. Sparse representation based on adaptive multiscale features for robust machinery fault diagnosis. Proceedings of the Institution of Mechanical Engineers, Part C: Journal of Mechanical Engineering Science, Vol. 229, Issue 12, 2015, p. 2303-2313.

[15] Xu S., Liu Y., Xu S. Wood defects recognition based on the convolutional neural network. Journal of Shandong University (Engineering Science), Vol. 43, Issue 2, 2013.

[16] Jarrett K., Kavukcuoglu K., Ranzato M., et al. What is the best multi-stage architecture for object recognition? IEEE 12th International Conference on Computer Vision, 2009.

[17] Kavukcuoglu K., Sermanet P., Boureau Y.-L., et al. Learning convolutional feature hierarchies for visual recognition. Advances in Neural Information Processing Systems, 2010.

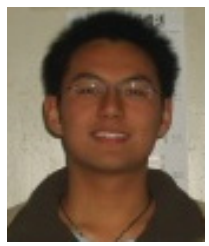

Jiyu Yan received his B.S. degree in College of Mechanical Engineering from Zhengzhou University, Zhengzhou, China, in 2010.Then he received his Master's degree in College of Field Engineering from PLA University of Science and Technology, Nanjing, China, in 2013. Now he is a Ph.D. candidate at PLA University of Science and Technology, China. His current research interests include signal processing, machinery fault diagnosis and vehicle terramechanics.

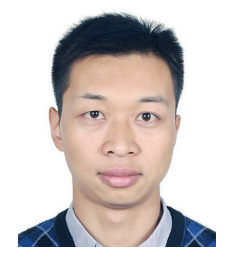

Huijie Zhu received his Ph.D. degree in mechanical electronic engineering from PLA University of Science and Technology, Nanjing, China. Now he works as an engineer in the first engineering scientific research institute, Wuxi, China. His current research interests include unmanned vehicle, machine learning and signal processing.

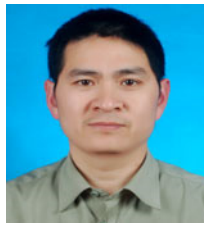

Xiaoqiang Yang received his M.S. and Ph.D. degrees from PLA University of Science and Technology, Nanjing, China, in 1992 and 2002, respectively. Since December 2001, he has been affiliated with the Department of Mechanic and Electronic Engineering at the Institute of Field Engineering, Nanjing, as an Associated Professor. His teaching and research interests are in the areas of signal processing, fault diagnosis and virtual instrumentation technology. 


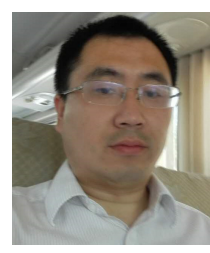

Youhui Cao received his Ph.D. degree in vehicular engineering from Academy of Armored Force Engineering in 2008. Now he works as a vice-senior engineer in the first engineering scientific research institute, Wuxi, China. His current research interests are electronic control and unmanned vehicle.

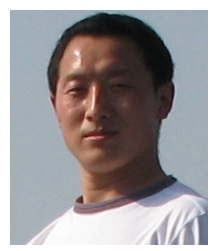

Lifu Shao received his M.S. degree in mechanical design and theory from PLA University of Science and Technology in 2006. Now he works as an engineer in the first engineering scientific research institute, Wuxi, China. His current research interest is mechanical-electronic engineering and unmanned vehicle. 